\title{
A new parallel bat algorithm for musical note recognition
}

\author{
Ansam Nazar Younis, Fawziya Mahmood Ramo \\ Department of Computer Science, College of Computer Science and Mathematics, University of Mosul, Iraq
}

\begin{tabular}{l} 
Article Info \\
\hline Article history: \\
Received Jan 18, 2020 \\
Revised Jun 13, 2020 \\
Accepted Aug 5, 2020 \\
\hline Keywords: \\
Bat algorithm \\
Linear discriminate analysis \\
Metaheuristic \\
Musical notes \\
Parallel processing
\end{tabular}

\begin{abstract}
Music is a universal language that does not require an interpreter, where feelings and sensitivities are united, regardless of the different peoples and languages, The proposed system consists of two main stages: the process of extracting important properties using the linear discrimination analysis (LDA) This step is carried out after the initial treatment process using various procedures to remove musical lines, The second stage describes the recognition process using the bat algorithm, which is one of the metaheuristic algorithms after modifying the bat algorithm to obtain better discriminating results. The proposed system was supported by parallel implementation using the (developed bat algorithm DBA), which increased the speed of implementation significantly. The method was applied to 1250 different images of musical notes. The proposed system was implemented using MATLAB R2016a, Work was done on a Windows10 Processor OS (Intel @ Core TM i5-7200U CPU @ 2.50GHZ 2.70GHZ) computer.
\end{abstract}

This is an open access article under the CC BY-SA license.

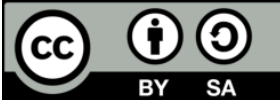

\section{Corresponding Author:}

Ansam Nazar Younis,

Department of Computer Science,

University of Mosul,

Mosul, Iraq.

Email: ANSAM201733@yahoo.com

\section{INTRODUCTION}

Music is a kind of world art that deals with the composition, rhythm and distribution of various melodies. Music is a science that explores melodies' origins and principles in terms of harmony and distinction a language that includes expressive and communicative components. It is an art composed over certain periods of time with sounds and silence [1]. As music is a general process known to those interested in the music industry, the computer had to be used to serve the music Several computer scientists have developed programs, systems and algorithms to recognize and discover various musical notes and applications for music [2].

The pattern distinction refers to the function of putting a particular object in the appropriate distinction based on that object's parameters, Usually done automatically with the aid of a computer after the object's properties have been extracted. The term pattern recognition includes several other important terms such as recognition, description, classification, and grouping of patterns. For example, a pattern may be a fingerprint image, a handwritten word, a human face, or a speech signal [3].

In the year (2011), the two researchers (Joyce Ooi Boon Ee and Alan WC Tan) use box bounding and template matching [4]. In (2014) the two researchers (Yoppy Sazaki and Rosda Ayuni) use the Minimum Spanning Tree algorithm and the Euclidean distance [5]. In (2017) the researchers (Jan Hajic c and Pavel Pecina) suggested to use convolutional neural network CNN for discover the head's notes applied to various patterns of handwriting styles [6]. In (2018) Ansam Nizar and Fawzia Mahmoud proposed a method for recognize musical notes using the algorithm (LDA linear discriminate analysis) and an equation of similarity scale index SSIM [7]. 
The goal of the research is to develop and create an intelligent computer system to recognize images of musical notes and to classify their form in order to help spread musical culture through different segments of society and to ensure simplicity, transparency, accuracy and speed in communicating with users at the same time. The research further aims to teach the computer and apply knowledge to it in the field of music education and to set up a supportive system for music experts to learn musical notes and provide a system for those wanting to learn the meaning of musical scale symbols.

\section{COMMON NOTATION}

The music is written in letters that are different from the alphabet, Music notes are a time-bound symbol, depending on the sound of that letter, the duration of each letter or musical symbol on the staff $[1,8]$ note Figure 1.

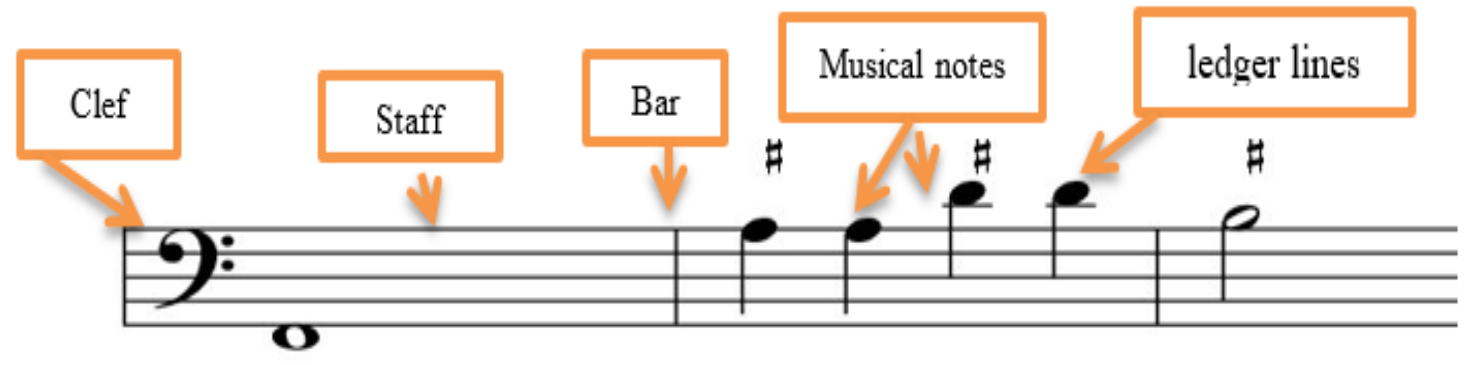

Figure 1. Staff lines

\subsection{The staff}

Five horizontal lines, including four equal spaces, are parallel and equal in length. Some musical notes are positioned on or at a distance between lines. Additional short lines may be attached to the ledger lines to represent a very high or very low mark that is not in standard condition [8].

\subsection{Musical notes}

It is composed of seven simple musical signs of different sound and the eighth sound is a repetition of the first note: du-ri-mi-fa-la-si then du again. The name of the music note is determined by the key used at the beginning of the music scale, The note form is an oval head that can be complete and empty, and the head may be attached to the stem It may also be linked to one or more flags (flag) This depends on the number of strikes and the length of the note [2]. Many musical notes affect the musical tone in one way or another, and each type of these symbols is written differently from the other and put on the musical scale in different positions and these symbols are:(clef, Accidental symbols, Rests, Time symbols, Breaks, Dynamics symbols, Augmentation Dot, Ties, Articulation, Signs Repeat, Economical symbols, Key Signature, Triad) [9].

\section{BAT ALGORITHM BA}

The Bats algorithm is one of the metaheuristic algorithms created by the world (Yang) in 2010, an algorithm inspired by nature [10]. Based on the previous features, The following three basic rules were developed for the algorithm: Bats use distance sensing echolocation and "learn" the difference between food, prey and backbones, In position $\mathrm{Xi}$, the bat flies at velocity (Vi) with the qmin frequency, with the variable wavelength $\lambda$ and the loudness L0 to hunt for the prey. It can automatically adjust the wave length (or frequency) from its emitted pulse and adjust the pulse emission rate $(0,1)$ according to its proximity to the target. Although the loudness may differ from one to another in many respects, it is assumed that the loudness is usually limited and that L0 varies from a large fixed value to a minimum constant value of Lmin [11].

Bat Algorithm steps [12]:

1) Determine the target function so that it represents:

$$
f(X), X=(x 1, \ldots, x d)^{T}
$$


2) Configure the bat population:

$$
X_{i}(i=1,2,3, \ldots n)
$$

where $\mathrm{n}$ is the maximum number of bats, then determines the initial velocity for each bat (Vi).

3) Define pulse frequency (qi ) at Xi location.

4) Configure pulse rates (ri)and loudness rate ( $\mathrm{Li})$.

5) Repeat the following steps until the condition is met (getting the maximum of the duplicates).

Create new solutions by adjusting frequency and updating speeds and locations solutions ((1) to (3)).

a) Compare the pulse frequency value with a random value.

- Select one of the best solutions.

- Create a local solution about the best solution selected.

b) Generate a new solution through the random flight of bats.

c) Compare the loudness value with the random value and compare the value of the new site with the value of the old site.

d) Accept new solutions.

e) Increase the (ri )pulse value and decrease the $\mathrm{Li}$ (loudness value).

f) Arrange the bat and find the best $x^{*}$ current.

6) Processing the results of the attached treatment and presentation.

The virtual bat movement is by simulating the virtual bat movement naturally. We have three basic rules where $\mathrm{Xi}$ and $\mathrm{Vi}$ are determined in d-dimensional and how to update it. The new solutions $\mathrm{Xi} t$ and velocities Vit at time $t$ are given using the following equations [13]:

$$
\begin{aligned}
& q_{i}=q_{\text {min }}+\left(q_{\text {max }}-q_{\text {min }}\right) \beta \\
& V_{i}^{t}=V_{i}^{t-1}+\left(X_{i}^{t}-X_{*}\right) q_{i} \\
& X_{i}^{t}=X_{i}^{t}+V_{i}^{t}
\end{aligned}
$$

$\beta \epsilon(0,1)$ is a random vector taken from a regular distribution. $X^{*}$ represents the best current general location (solution) which is located after comparing all the solutions among all $\mathrm{n}$ bats. For the local search segment. Once a solution has been identified among the best current solutions, a new solution for each bat is created locally using random walking as in (4):

$$
X_{n e w}=X_{o l d}+R L^{t}
$$

$\mathrm{R} \epsilon(-1,1)$ is a random number, $\mathrm{Lt}=(\mathrm{Lit})$ is the mean of loudness of all Bat in this time step [13]. The Li loudness and the pulse emission rate must be updated with repetition, according to the following equations:

$$
\begin{aligned}
& L_{i}^{t+1}=\alpha L_{i}^{t} \\
& r_{i}^{t+1}=r_{i}^{0}[1-\exp (-\gamma t)] \\
& L_{i}^{t} \rightarrow 0, r_{i}^{t} \rightarrow r_{i}^{0} \text { as } t \rightarrow \infty
\end{aligned}
$$

$\alpha$ and $\gamma$ are constant values. For each: $0<\alpha<1$ and $\gamma>0$.

$$
L_{i}^{t} \rightarrow 0, r_{i}^{t} \rightarrow r_{i}^{0} \quad \text { and } t \rightarrow \infty
$$

The selection of parameters requires some experimentation. Initially, each bat must have different values for the loudness rate and the pulse emission rate. This can be achieved by random distribution [14]. 


\section{FEATURE EXTRACTION}

Feature extraction methods are used in the process of dimensional reduction of the original highdimensional data and are stronger than the methods of character selection [7, 15]. The process of extracting attributes represents the process of creating a new set of features that are more important by mixing the original features in linear or nonlinear ways $[16,17]$. Linear discriminate analysis (LDA) is a commonly supervised modality (ie taking classification into account) And is commonly used in computer vision, pattern recognition, machine learning and the like, also known as Fisher's linear discriminator. To the British statistical world (Ronald Aylmer Fisher), who initially proposed the approach and is an effective method of discrimination, since it is a linear method of linear data reduction and a classification method [18]. LDA attempts to define a new axis to reduce inter-class variation due to session/channel effects and to maximize differences between classes [19]. The linear discrimination analysis algorithm aims at finding vectors that maximize the difference between the different classes (Between-Class Scatter Matrix CB), Reducing sample variability within each category Within-Class Scatter Matrix (CW) [18, 19].

\section{PARALLEL PROCESSING}

Parallel processing means faster program execution by splitting the program into multiple parts and performing at the same time as each part runs on a separate processor or on a separate kernel. The program implemented in $\mathrm{N}$ processors is approximately $\mathrm{N}$ faster than the same program on a single processor [20]. Its characteristics can be summarized as follows [21]:

- $\quad$ Uses more than one processing unit (CPUs).

- Divide the problem into separate parts can be solved synchronously.

- Divide each part into a series of prompts.

- The instructions from each part are carried out simultaneously on different processing units.

- All implementation is under control.

\subsection{Multicore processors}

This type of processors is also called the Chip Multiprocessor, combining two or more processors (called Cores) on one piece of silicon (called the mold). Each kernel consists of all components of an independent processor, such as Registers, ALU, Control Unit, As well as data storage (L1 data caches). In addition to multiple cores, contemporary multi-core chips also include L2 cache and L3 cache in some cases [22].

\section{DESIGN THE PROPOSED SYSTEM}

The proposed PBMRS (parallel bat musical notes recognition system) system was designed to build a smart computer program to recognize musical notes and to display useful information about each musical score. The proposed system goes through two phases: training and testing.

\subsection{Design of the training phase}

Building database of images: In this stage, read the 1000 musical notes images of the training phase of 100 different music labels. Preprocessing: Initial treatment includes the following steps:

a. Resize each image and unify the size of all images

b. converting images from color images (RGB) to gray colors (Gray)

c. Converting images from Gray color to Binary images (black and white), in order to reduce the number of colors, it is easy to distinguish the color of the mark from the background color.

d. Delete the white frame surrounding the musical mark and the background, thereby removing the important information to focus only on the shape of the mark.

e. Resize the image after the process of deleting the frame.

f. Feature extraction: find the matrix of weights for a musical mark model as a result of the linear discrimination analysis algorithm (LDA).

Recognition using the bat algorithm:

a. Intelligent bat algorithm developed: The traditional bats algorithm was developed by adding several steps to improve the results and their suitability to the discriminating process. The proposed algorithm was called the developed bat algorithm DBA which is consists of the following steps (steps added in bold):

1) Define the target function represented by the musical input.

2) Parameters configuration: Given the initial values and constant values of the parameters for implementing the algorithm as follows:

a) Determining the number of bats with the number of models of the musical notes.

b) Sets the velocities $\left(\mathrm{V}_{\mathrm{i}}\right)$ of the bats by defining a matrix that is equal in size musical notes.

c) Determine the pulse frequency $\left(\mathrm{Q}_{\mathrm{i}}\right)$ so that the frequency value is between $\mathrm{Q}_{\min }$ and $\mathrm{Q}_{\max }$. 
d) Determination of the $r_{i}$ pulse rates [0-1].

e) Select a value for loudness $\mathrm{Ai}$ as a random value within $(0,1)$, to be a constant value for all cycles.

f) Determine the number of iterations by the number of bats.

3) The weights matrix for the target function and for the current bat for each cycle are initialized as a new step supported by the algorithm and represent the weights derived from the linear discrimination analysis algorithm (LDA).

4) Find the fitness function for each matrix of bat weights for the weight matrix of the target function by using the correlation between the two matrices represented by the following formula [23]:

$$
\text { fitness }=\frac{\sum_{m} \sum_{n}\left(W_{m n}-\bar{W}\right)\left(B_{m n}-\bar{B}\right)}{\sqrt{\left.\sum_{m} \sum_{n}\left(W_{m n}-\bar{W}\right)\right)^{2} \sum_{m} \sum_{n}\left(\left(B_{m n}-\bar{B}\right)\right)^{2}}}
$$

$\mathrm{W}$ : represents the weight matrix of a bat whose size is $\mathrm{m}^{*} \mathrm{n}$. B: represents the weight matrix of the target function whose size is also $\mathrm{m}^{*} \mathrm{n}$,

$$
\bar{W}=\operatorname{mean} 2(A), \text { And } \bar{B}=\operatorname{mean} 2(B)
$$

Create new solutions by adjusting frequency and updating speeds and positions by the following equations:

$$
\begin{aligned}
& Q_{i}=Q_{\min }+\left(Q_{\max }-Q_{\min }\right) \beta \\
& V_{i}^{t}=V_{i}^{t-1}+\left(X_{i}^{t}-X_{*}\right) Q_{i} \\
& X_{i}^{t}=X_{i}^{t}+V_{i}^{t}
\end{aligned}
$$

As: $\mathrm{Q}_{\mathrm{i}} \quad$ : The new frequency of the bat $\mathrm{i}$.

$\mathrm{X}_{\mathrm{i}}^{\mathrm{t}-1} \quad$ : The value of the old solution.

$\beta \quad$ : A random value confined between duration $(0,1)$.

$\mathrm{V}_{\mathrm{i}}{ }^{\mathrm{t}} \quad$ : The amount of new velocity of the $\mathrm{i}$ in the current step $\mathrm{t}$.

$\mathrm{V}_{\mathrm{i}}^{\mathrm{t}-1} \quad$ : The amount of bat speed $\mathrm{i}$ in the previous step $\mathrm{t}-1$.

$\mathrm{X}_{\mathrm{i}}^{\mathrm{t}} \quad$ : The solution when the current step $\mathrm{t}$.

$\mathrm{X}_{*} \quad$ : Represents the best current bat that represents the best current site.

5) Balances the pulse rate with a random value to determine whether a solution can be identified among the best new solutions and create a local solution around the best solution selected.

6) Generate a new solution by random flight of the bat using the following equation. The amount of the step in random flight is determined as a random value (0.001). The fitness function is then calculated for the new solution for the target function.

$$
X_{\text {new }}=X_{\text {old }}+\varepsilon * 0.001
$$

As: $\mathrm{X}_{\text {new }}$ the value of the best new solution, $\mathrm{X}_{\text {old }}$ : Best value for old solution, $\varepsilon$ : random value $[-1,1]$.

7) Balance the fitness value of the new solution with the value of fitness for the old solution and compare the loudness with the random value to determine if the current bat is close to the prey better, then determine the acceptance of new solutions or not.

8) Check the stop condition (number of bat)? If the condition is met, it will continue, otherwise it will be referred to step 5 .

9) Arrange bats and find the best $X *$ current, by finding the largest current fitness function.

10)Find the best solution site, which represents the best bat site.

11)Display results.

b. Parallel bats implementation algorithm: a parallel-developed bats algorithm with a single multi-core processor was suggested. Parallel programming model used for this purpose is SPMD model. Each core performs one program in the processor at one time on different datasets. This model is based on a type of parallel programming model, the master-slave Model. Parallel method used in this developed bat algorithm was the algorithm-level. 


\subsection{Design of the test phase}

The test database was created from a collection of different musical tag images. Different models of the training models are created from each type of musical label, as well as the creation of models that include the musical lines and the creation of models that are strange to the music labels. In the folder (Testing) $500(300+200)$ different image of 100 different types of musical labels, as well as 50 other strange images.

\section{EVALUATION SYSTEM PERFORMANCE MEASURES}

a. Detection rate DR: The ratio of the recognizing of music notes in the system is correct.

b. True negative TN: The number of images of wrong musical notes, and recognized wrong.

c. False positive rate FPR: The number of wrong musical notes images and recognized correct.

d. True positive rate TPR: It represents the number of correct music notes, and is properly categorized.

e. False negative FN: It represents the number of correct music notes, and is properly wrong categorized.

f. Sensitivity: Measures the ability of the discriminating system to find images of wrong musical notes.

g. Specificity: Measures the ability of the discriminating system to find images of correct musical notes.

h. False rejection ratio FRR: The proportion of wrongly tagged music images (not found).

i. Wrong acceptance ratio WRR: The proportion of wrongly accepted music scores (Wrong result).

The following equations can be calculated from line ratios above [24]:

a. Detection rate $\mathrm{DR}=($ Number of sample correctly detected $) /($ Total number of samples $) * 100$.

b. Sensitivity $=\mathrm{TPR} /(\mathrm{TPR}+\mathrm{FN}) * 100 \%$.

c. Specificity $=\mathrm{TN} /(\mathrm{TN}+\mathrm{FPR}) * 100 \%$.

d. False negative rate $(\mathrm{FN} \%)=\mathrm{FN} /(\mathrm{TPR}+\mathrm{FN})=100 \%$-Sensitivity.

e. False positive rate $(\mathrm{FPR} \%)=\mathrm{FPR} /(\mathrm{TN}+\mathrm{FPR})=100 \%$-Specificity

f. Positive predictive rate $(\mathrm{PP} \%)=\mathrm{TPR} /(\mathrm{TPR}+\mathrm{FPR}) * 100 \%$.

g. Negative predictive rate $(\mathrm{NPR} \%)=\mathrm{TN} /(\mathrm{TN}+\mathrm{FN}) * 100 \%$.

h. False rejection rate $(\mathrm{FRR})=($ Number of images rejected error $) /($ Total number of images $) * 100 \%$.

i. Wrong acceptance rate $(\mathrm{WRR})=($ Number of images accepted error $) /($ Total number of images $) * 100 \%$.

\section{IMPLEMENTATION OF THE PROPOSED ALGORITHM}

Implementation were conducted and the proposed PBMRS system was tested to identify the different musical notes in two ways (deleting the musical lines and not deleting the musical lines) and using two types of implementation methods (sequential and parallel).

\subsection{System training and testing stage}

The data were trained in the proposed PBMRS system consisting of 700 images. The results of the training was obtained according to the evaluation measures, where FN, FPR, FRR and WRR equal to $0 \%$. The value of DR, NPR, PP, Sensitivity and Specificity is $100 \%$. The proposed PBMRS algorithm was tested on a collection of 300 images of musical notes.

\subsection{Recent experiences}

200 new images were added to the images of the notes, the first section contains the staff lines and another section is free from the lines, and a group of exotic images (50 pictures), which represent images of some shapes and symbols, are added to the musical notes. Image the test results were as shown in the Table 1.

Table 1. Results of the system in the testing stage

\begin{tabular}{ccc}
\hline Type of criterion & $\begin{array}{c}\text { Result by way of deleting } \\
\text { staff lines }\end{array}$ & $\begin{array}{c}\text { Result in a way that the staff } \\
\text { lines are not deleted }\end{array}$ \\
\hline FN & $2.67 \%$ & $13.5 \%$ \\
FPR & $0 \%$ & $8 \%$ \\
DR & $97.34 \%$ & $86.5 \%$ \\
NPR & $100 \%$ & $63.014 \%$ \\
PP & $100 \%$ & $97.75 \%$ \\
Sensitivity & $97.34 \%$ & $86.5 \%$ \\
Specificity & $100 \%$ & $92 \%$ \\
FRR & $1 \%$ & $11.5 \%$ \\
WRR & $1.67 \%$ & $2 \%$ \\
\hline
\end{tabular}




\section{CRITERIA FOR INCREASING SYSTEM SPEED AND EFFICIENCY $[25,26]$}

$$
\begin{aligned}
& \text { Speed Up }=\frac{\text { The execution time of serial execution(one core })}{\text { The execution time on } n \text { cores }} \\
& \text { Speed up Efficiency }=(\text { Speed } U p) / n
\end{aligned}
$$

$\mathrm{n}$ : represents the number of cores used in the implementation, the system was implemented in a sequential and parallel manner using two and three and four cores, and the results indicated in the Table 2 were obtained:

Table 2. Results of serial implementation and parallel implementation

\begin{tabular}{ccccc}
\hline No. of cores & $\begin{array}{c}\text { Result by way of deleting staff lines } \\
\text { Speed Up }\end{array}$ & $\begin{array}{c}\text { Result in a way that the staff lines are not deleted } \\
\text { Speed up Efficiency }\end{array}$ & $\begin{array}{c}\text { Speed Up } \\
\text { Speed up Efficiency }\end{array}$ \\
\hline 2-cores & 1 & 1 & 1 & 1 \\
3-cores & 1.61688 & 0.80844 & 1.5615 & 0.7808 \\
4-cores & 2.371 & 0.651253 & 1.822 & 0.6071 \\
\hline
\end{tabular}

\section{DISCUSSION}

It was noted in Table 2 that the percentage of discrimination reached $95.5 \%$ by deleting musical lines, while $86.5 \%$ by not deleting those lines. The use of the process of deleting music lines is the best way to obtain clearer images and thus get higher results of discrimination and standards at better rates. It was observed in Table 2 that the use of three cores and four cores achieves a higher speed than two cores, although the use of three core and four core achieves a higher acceleration factor than the use of two core in the implementation. However, the use of two core is the most efficient, especially the use of the method of deleting the lines of the musical scale as the efficiency of the speed of the system about $(0.80844)$ because the size of data used in each nucleus in the implementation of the use of two core is larger than the size of data used in each core in the three-cores and four implementation. A comparison was made between the results of the proposed PBMRS system and the results of a number of researchers in the same field as shown in Table 3.

\begin{tabular}{|c|c|c|c|c|c|}
\hline No. & Research name & Algorithm & $\begin{array}{l}\text { Number of } \\
\text { Musical Notes }\end{array}$ & $\begin{array}{l}\text { Test models } \\
\text { number }\end{array}$ & $\begin{array}{l}\text { Recognition } \\
\text { Rate }\end{array}$ \\
\hline 1 & Music Symbol Recognition/2011 & Template Matching & 7 & 585 & $94.359 \%$ \\
\hline 2 & $\begin{array}{l}\text { Musical Note Recognition Using } \\
\text { Minimum Spanning Tree } \\
\text { Algorithm/2014 }\end{array}$ & $\begin{array}{l}\text { Minimum Spanning } \\
\text { Tree\& Euclidean distance }\end{array}$ & 7 & 97 & $97.45 \%$ \\
\hline 3 & $\begin{array}{l}\text { Detecting Note heads in Handwritten } \\
\text { Scores with Conv. Nets and } \\
\text { Bounding Box Regression/2017 }\end{array}$ & $\begin{array}{l}\text { CNN Convolutional } \\
\text { Neural Network }\end{array}$ & 7 & 140 & $96 \%$ \\
\hline 4 & $\begin{array}{l}\text { Distinguish Musical Symbol Printed } \\
\text { using the Linear /Discriminant } \\
\text { Analysis LDA and Similarity } \\
\text { Scale/2018 }\end{array}$ & $\begin{array}{l}\text { Linear Discriminant } \\
\text { Analysis LDA\& } \\
\text { Similarity Scale SSIM }\end{array}$ & 15 & 180 & $89.5 \%$ \\
\hline 5 & $\begin{array}{l}\text { A New Parallel Bat Algorithm For } \\
\text { Musical Note Recognition/2020 }\end{array}$ & $\begin{array}{l}\text { Bat Algorithms } \\
\text { (Sequential execution, } \\
\text { Parallel execution) }\end{array}$ & 100 & 1250 & $97.34 \%$ \\
\hline
\end{tabular}

Table 3. A comparison of results with other algorithms

\section{CONCLUSION}

The extraction of properties using LDA algorithm helped reduce the reservoir capacity of the images and reduce the implementation time of the system. After the size of the images used about $1000 * 625$, it became a matrix of characteristics with two dimensions about $625 * 625$. Also choose a small constant random value represented by the value $(0.001)$ which is used to determine the amount of step (flight distance) of the bat. It is an important step added to the algorithm because the step amount in the bat is usually large because it is waiting for echo before deciding the type of target and determining its destination. Determining the amount of stock step reduces the unnecessary large dimensions that do not fit their use. So the percentage of discrimination in the manner of deleting the lines was equal to $(96.6 \%)$ and the percentage of discrimination by way of non-deletion of lines equal to $(93 \%)$. 
As a future works: Adding the method for principle components analysis (PCA), then using the linear discrimination analysis method to obtain stronger characteristics of the images, and thus obtain better discrimination, The use of a greater number of image models of musical notes, which strengthens the process of distinguishing and benefiting from the advantages of parallel implementation in a more efficient manner, such as adding handwritten musical signs, and analyze the musical notes placed on the staff by determining their position on the staff lines and what tone they produce based on the Clef.

\section{ACKNOWLEDGEMENTS}

The authors are very grateful to the university of Mosul/College of computer Science and Mathematics for their provided facilities, which helped to improve the quality of this work.

\section{REFERENCES}

[1] B. Hansen, et al., "Introduction to Music Appreciation," ePress Course Materials, American Public University System, 2014.

[2] S. J. Mohammed, "Standard printed musical note recognition based on neural network," Journal of University of Anbar for Pure Science, vol. 6, no. 2, pp. 20-25, 2012.

[3] J. Tohka, "SGN-2506: Introduction to Pattern Recognition," Tampere University of Technology, 2006.

[4] J. O. B. Ee and A. W. C. Tan, "Music Symbol Recognition," Symposium on Information \& Computer Sciences (ICS 2011), pp. 1-4, 2011.

[5] Y. Sazaki, et al., "Musical note recognition using Minimum Spanning Tree Algorithm," 2014 8th International Conference on Telecommunication Systems Services and Applications (TSSA), Kuta, pp. 1-5, 2014.

[6] J. Haji`c and P. Pecina, "Detecting Noteheads in Handwritten Scores with ConvNets and Bounding Box Regression," arXiv: 1708.01806v1, 2017.

[7] A. N. Younis and F. M. Remo, "Distinguish Musical Symbol Printed using the Linear Discriminant Analysis LDA and Similarity Scale," International Journal of Computer Applications, vol. 179, no. 47, pp. 20-24, 2018.

[8] C. Schmidt-Jones, "Reading Music: Common Notation," CONNEXIONS, Rice University, 2008.

[9] J. Harnum, "Baisc music theory: how to read, write, and understand written music," Sol-Ut Press, 2001.

[10] X.-S. Yang, "A New Metaheuristic Bat-Inspired Algorithm," in: Nature Inspired Cooperative Strategies for Optimization (NISCO 2010) (Eds. J. R. Gonzalez et al.), Studies in Computational Intelligence, Springer Berlin, 284, Springer, 65-74, 2010.

[11] El-Ghazali T., "Metaheuristics from Design to Implementation," Wiley, 2009.

[12] Y. Zhou, et al., "A Novel Hybrid Bat Algorithm for the Multilevel Thresholding Medical Image Segmentation," Journal of Medical Imaging and Health Informatics, vol. 5, no. 8, pp. 1742-1746, 2015.

[13] X.-S. Yang and X. He, "Bat Algorithm: Literature Review and Applications," International Journal of Bio-Inspired Computation, vol. 5, no. 3, pp. 141-149, 2013.

[14] A. M. Taha, et al., "Naive Bayes-Guided Bat Algorithm for Feature Selection," The Scientific World Journal, vol. 2013, pp. 1-9, 2013.

[15] I. Guyon and A. Elisseeff, "An Introduction to Variable and Feature Selection," Journal of Machine Learning Research, vol. 3, pp. 1157-1182, 2003.

[16] S. Cateni, et al., "Variable Selection and Feature Extraction through Artificial Intelligence Techniques," Multivariate Analysis in Management, Engineering and the Sciences, InTech, 2013.

[17] A. Eleyan and H. Demirel, "PCA and LDA based neural networks for Human Face Recognition," in K. Delac and M. Grgic (eds), "Face Recognition," I-Tech Education and Publishing, Vienna, Austria, pp. 93-106, 2007.

[18] C. Bishop, "Pattern Recognition and Machine Learning," Springer Science+Business Media, LLC, 2006.

[19] S. Singh, "Bayesian distance metric learning and its application in automatic speaker recognition systems," International Journal of Electrical and Computer Engineering (IJECE), vol. 9, no. 4, pp. 2960-2967, 2019.

[20] B. Basil, "New Platform for Mobile Cloud Computing," M.Sc. Thesis, University of Mosul/Computer and Mathematics College, 2017.

[21] B. Barney, "Introduction to Parallel Computing," Lawrence Livermore National Laboratory, 2010.

[22] W. Stallings, "Computer Organization and Architecture Designing for Performance," Eighth Edition, Prentice Hall Upper Saddle River, 2010.

[23] R. C. Gonzalez and R. E. Woods, "Digital Image Processing Third Edition," Pearson Prentice Hall, 2008.

[24] A. Nazar, "Developing and Implementation of Metaheuristic Algorithm Sequentially and Parallel for Musical Notes Recognition," M. Thesis, Department of Computer Science, College of Computer and Mathematical Sciences, University of Mosul, 2018.

[25] P. Pacheco, “An Introduction to Parallel Programming," Elsevier Inc, 2011.

[26] P. Salza and F. Ferrucci, "Speed up genetic algorithms in the cloud using software containers," Future Generation Computer Systems, vol. 92, pp. 276-289, 2019. 


\section{BIOGRAPHIES OF AUTHORS}

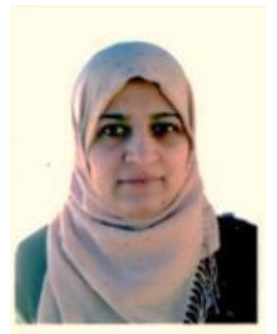

Ansam Nazar Younis She has been an assistant literature at department of computer sciences, college of computer sciences and mathematics, the University of Mosul, Iraq since 2018, Graduated from the Computer Science and Mathematics Collage at the University of Mosul, Iraq in 2005, and worked as a programmer in the same collage until 2013 when she also started studying Masters of Science in the same collage, then she finished MSC. Degree at 2018. General expertise is computer science, and specialty is in the area of artificial intelligence and image processing. She is this research's co-author. She has a research gate account under the name Ansam Nazar. Email is: anyma8@uomosul.edu.iq

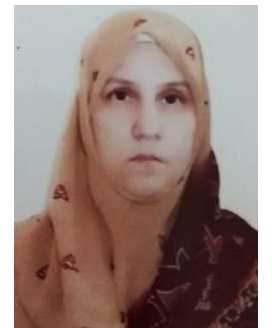

Fawziya Mahmood Ramo Obtained BA degree in Computer Science in 1992, then obtained MA degree in Computer Architecture in 2001 and PhD in Artificial Intelligence in 2007, obtained Assistant Professor in 2013, interested by research in computer science and artificial intelligence and machine learning. email: fmrmb7@yahoo.com 